\title{
Study of Clinical and Biochemical Parameters of Type 2 Diabetic Subjects with Pulmonary Tuberculosis
}

\author{
AFSANA Fa ${ }^{\mathrm{a}}$, JAMIL SNAA ${ }^{\mathrm{b}}$, LATIF ZA ${ }^{\mathrm{b}}$
}

\begin{abstract}
:
Aims: Type $2 \mathrm{DM}$ is a global epidemic and recognized as a threat to pulmonary tuberculosis (PTB) control worldwide especially in developing countries. When tuberculosis is diagnosed in diabetic subjects both can be affected in term of clinical presentation and course of disease. The aim of the study was to evaluate the demographic, clinical and biochemical parameters of newly detected PTB patients with type 2 diabetes.
\end{abstract}

Methods: Seventy two diabetic subjects with newly detected PTB attending outpatient department, BIRDEM were studied. Patients with fever, cough, hemoptysis and/or weight loss were interviewed by a structured questionnaire. After thorough clinical examination, blood sugar, complete blood count (CBC), ESR, sputum for bacteriological culture and acid fast bacilli (AFB) (3 samples), $X$-ray chest were done. The diagnosis of PTB was based on a positive sputum AFB test, a suggestive $C B C$ report or typical radiographic findings with high clinical probability. Diagnosed PTB cases were included in the study. All patients were followed up at least at1st, $3^{\text {rd }}$ and $6^{\text {th }}$ month of antitubercular therapy.

Result: Mean age of study subjects was 46(19-75) years. The study subjects do not have past history of tuberculosis. Most

Introduction:

Incidence of tuberculosis remains high in low socioeconomic countries that have high rates of infection, high prevalence of malnutrition and crowded living conditions, or poor tuberculosis control

a. Dr. Faria Afsana, Registrar, Dept. of Endocrinology, Bangladesh, Institute of Research, Rehabilitation of Endocrine and Metabolic Disorder (BIRDEM), Dhaka, Bangladesh

b. Dr. S.N.A. Ashraf Jamil, DCMO, Medicine OPD, BIRDEM, Dhaka, Bangladesh,

c. Prof. Zafar Ahmed Latif, Prof. and Head Dept. of Endocrinology, BIRDEM, Dhaka, Bangladesh.

Address of Corespondence to : Dr. Faria Afsana, Registrar, Dept. of Endocrinology ,Bangladesh Institute of Research, Rehabilitation of Endocrine and Metabolic Disorder (BIRDEM), Dhaka, Bangladesh.

Received: March 28, 2013

Accepted: December 31, 2013 of the study subjects (98.6\%) were on insulin for treatment of diabetes. Mean body mass index (BMI) was $19.9 \mathrm{~kg} / \mathrm{m}^{2}$. Mean ESR ( $\mathrm{mm}$ in $1^{\text {st }}$ hour) was 94.5 with $60 \%$ subjects having ESR $>100$. Sputum for bacteriological culture revealed no growth in $77.3 \%$ patients. Chest $X$-ray revealed cavity in $47.2 \%$, opacity in $40.3 \%$, both opacity and cavity in $5.6 \%$, pleural effusion in $5.6 \%$ and $5.5 \%$ had no detectable lesion. All patients were sputum AFB positive and among them $73.6 \%(n=53)$ had AFB positive in all 3 samples. Most of the patients ( $n=62,86.6 \%$ ) become sputum $A F B$ negative after one month of treatment initiation. Rest 10 subjects (13.4\%) become sputum negative in 2 months follow up and all of these patients had cavitary lesions in chest $X$-rays. A good number of the patients (98\%) with positive $x$-ray finding showed radiological improvement after 2 months of antitubercular treatment. All the patients completed their therapy without any interruption for 6 months.

Conclusion: Pulmonary cavity and opacity is the commonest radiological finding among study subjects. Sputum positivity for AFB is a good diagnostic tool for PTB in diabetic subjects. Subjects with cavitary lesion in chest $X$-ray become sputum negative than others.

Keywords: Pulmonary Tuberculosis, Diabetes, Sputum AFB

(Birdem Med J 2014; 4(1): 5-8)

infrastructure. At the same time, prevalence of diabetes is increasing globally in an epidemic fashion. There is growing evidence that diabetes mellitus is an important risk factor for tuberculosis and might affect disease presentation and treatment response. Furthermore, tuberculosis might worsen glycaemic control in people with diabetes. The association between diabetes mellitus and tuberculosis and their synergistic role in causing human disease has been recognised for centuries. In recent decades, tuberculosis has increasingly become a problem in low-income countries, particularly those with changing patterns of diet and physical activity, and aging populations. ${ }^{1,2}$ The effect of diabetes on the development and severity of tuberculosis, and the complex interrelations between nutrition, obesity, 
diabetes, and tuberculosis remain provocative issues in public health and clinical medicine. ${ }^{3,4}$ In the setting of the increasing overlap of populations at risk for both diseases, the combination of tuberculosis and diabetes mellitus represents a worldwide health threat. Noncommunicable diseases, which represented $47 \%$ of the disease burden in 1990 in low-income countries, have been predicted to rise to $69 \%$ by $2020 .{ }^{5}$ In the past 20 years, the debate over whether diabetes mellitus causes increased susceptibility to tuberculosis, as well as differences in presentation, severity, and response to therapy, has been rekindled. The risk of developing active tuberculosis is a two-step process, beginning with initial exposure to and infection by Mycobacterium tuberculosis followed by subsequent progression to disease. Studies of diabetes mellitus and tuberculosis generally focus on active tuberculosis disease. Indeed, a recent large meta-analysis showed that diabetic patients were $3 \cdot 1$ times (95\% CI 2·27-4·26) more likely to have tuberculosis than controls, with higher effect sizes in non-North American populations. ${ }^{6}$ In a recent study of diabetic subjects in Hong Kong, those with haemoglobin A1c greater than 7\% had a three times increased hazard of active tuberculosis compared with those with haemoglobin A1c less than 7\%. ${ }^{7}$ These data suggest that poor glycaemic control is a risk factor for tuberculosis. Poorly controlled diabetes can lead to multiple complications, including vascular disease, neuropathy, and increased susceptibility to infection. ${ }^{8}$ Diabetes might also lead to increased susceptibility to disease caused by $M$ tuberculosis via multiple mechanisms. The mechanisms include those directly related to hyperglycaemia and cellular insulinopenia, as well as indirect effects on macrophage and lymphocyte function, leading to diminished ability to contain the organism.The radiographic presentation of tuberculosis depends on many factors, including duration of illness and host immune status. It was widely believed that pulmonary tuberculosis in diabetic patients presented with an atypical radiographic pattern and distribution, particularly lower-lung involvement. Also, patients with pulmonary tuberculosis that do not have upper-lobe involvement are less likely to have positive sputum smears and cultures. ${ }^{9}$ If diabetes alters immunity to tuberculosis, leading to higher baseline mycobacterial burdens and longer times to culture conversion with treatment, a higher rate of relapse might result. In studies assessing time to sputum-culture conversion, diabetic patients seem to take longer to achieve culture negativity. In one study in Turkey, patients with diabetes who received tuberculosis treatment had longer sputumculture conversion times than non-diabetic patients. ${ }^{10}$

\section{Material and Methods:}

Patients referred to pulmonologist from outpatient department, BIRDEM having symptoms of pulmonary tuberculosis (PTB) were selected and investigated purposively. Diagnosed PTB cases were included in the study. Seventy two diabetic subjects with newly detected PTB attending outpatient department, BIRDEM were studied. Patients with fever, cough, and hemoptysis and / or weight loss were interviewed by a structured questionnaire. After thorough clinical examination, blood sugar, complete blood count (CBC), ESR, sputum for bacteriological culture and acid fast bacilli (AFB) (3 samples), X-ray chest were done. The diagnosis of PTB was based on a positive sputum AFB test, a suggestive CBC report or typical radiographic findings with high clinical probability. All patients were followed up at least at $1^{\text {st }}, 3^{\text {rd }}$ and $6^{\text {th }}$ month of antitubercular therapy.The study was started in July 2010and 72 patients were enrolled within 3 months and each patient was followed up for at least 6 months from the beginning to end of the antitubercular therapy. Subjects included in this study were $>18$ years aged diabetic subjects with clinical features and/or radiological abnormalities consistent with PTB, sputum for AFB positive subjects. Subjects $<18$ year of age and PTB without DM were excluded from the study.Statistical Analysis was performed using SPSS (statistical Package for Social Science) 16 version. All data were expressed as mean, standard deviation (SD), range, percentage.

\section{Results:}

Mean age of study subjects was 46(19-75) years .Among them 46 (63.9\%) were male. Past history of contact with tuberculosis was absent among 71(98.6\%) subjects and none had past history of tuberculosis. Of the total study subjects, 48(66.7\%) were sedentary worker, 07(9.7\%) were day laborer and 17(23.6\%) were housewife. Most of the study subjects (98.6\%) were on insulin for treatment of diabetes. Duration of diabetes was less than 1 year in 33(45.8\%), 1-5 years in 13(18.1\%) and more than 5 years in 13(18.1\%) subjects. Mean BMI was $19.9 \mathrm{~kg} / \mathrm{m} .^{2}$ (Table-I). 
Table-I

Demographic characteristics of study subjects.

Variable

\begin{tabular}{lc}
\hline Mean Age (yrs ) & $46(19-75)$ \\
Sex (M) & $46(63.9 \%)$ \\
No H/O TB contact & $71(98.6 \%)$ \\
No P/H TB & $70(97.2 \%)$ \\
Occupation & \\
$\quad$ Sedentary worker & $48(66.7 \%)$ \\
$\quad$ Day labourer & $07(9.7 \%)$ \\
$\quad$ Housewife & $17(23.6 \%)$ \\
Treatment of Diabetes & \\
$\quad$ Insulin & $71(98.6 \%)$ \\
$\quad$ OAD & $1(1.4 \%)$ \\
Duration of diabetes & \\
$\quad<1 \mathrm{yr}$ & $33(45.8 \%)$ \\
$\quad 1-5 y r$ & $13(18.1 \%)$ \\
$>5 y r s$ & $26(36.1 \%)$ \\
BMI(mean $\pm S D)$ & $19.90 \mathrm{~kg} / \mathrm{m}^{2}( \pm 3.02)$ \\
\hline
\end{tabular}

Biochemical profiles showed that mean ESR ( $\mathrm{mm}$ in $1^{\text {st }}$ hour) was 94.5 with $60 \%$ subjects having ESR $>$ 100.Mean fasting and post prandial blood sugar (mmol/ l) were $13.69 \& 21.02$ respectively. (Table-II).

Table-II

Hematological and Biochemical profile of study
subjects.

\begin{tabular}{lc} 
Variables & Mean \pm SD \\
\hline Haemoglobin & $12.30( \pm 1.43)$ \\
Differential count of neutrophil $(\%)$ & $69.65( \pm 8.3)$ \\
Differential count of lymphocyte $(\%)$ & $22.14( \pm 9.63)$ \\
ESR(mm in $1^{\text {st }}$ hour $)$ & $94.53( \pm 36)$ \\
Fasting Blood glucose $(\mathrm{mmol} / \mathrm{l})$ & $13.69( \pm 4.71)$ \\
Postmeal Blood glucose $(\mathrm{mmol} / \mathrm{l})$ & $21.02( \pm 7.63)$ \\
\hline
\end{tabular}

Sputum for bacteriological culture revealed no growth in $77.8 \%$ patients, $12.5 \%$ had Klebsiella, $2.8 \%$ Pseudomonus \& candida each and Staphylococcus, Candida nonalbicans and Streptococcus 1.4\% each (Table-III). Chest X-ray revealed cavity in $47.2 \%$, opacity in $40.3 \%$, both opacity and cavity in $5.6 \%$, pleural effusion in $1.4 \%$ and $5.5 \%$ had no detectable finding (Figure 1).

Table-III

\section{Growth of organism in sputum of study subjects}

\begin{tabular}{lc} 
Name of organism & $\mathrm{N}(\%)$ \\
\hline Candida albicans & $2(2.8)$ \\
Klebsiella & $9(12.5)$ \\
Candida Nonalbicans & $1(1.4)$ \\
Pseudomonas & $2(2.8)$ \\
Stap. Aureus & $1(1.4)$ \\
Streptococcus & $1(1.4)$ \\
No growth & $56(77.8)$ \\
\hline
\end{tabular}

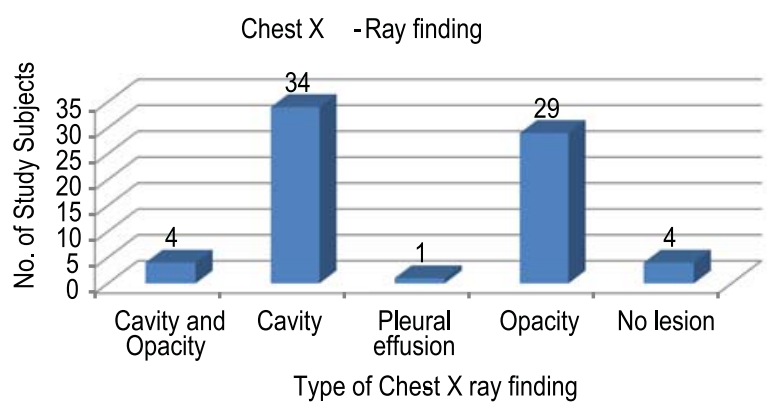

Fig.-1: Chest $X$-Ray findings of study subjects

All patients were sputum AFB positive and among them 73.6\% ( $n=53)$ had AFB positive in all 3 samples, 16.7\% $(n=12)$ had AFB positive in 2 samples and 9.7\% $(n=7)$ in one sample (Table-IV). Sixty two (86.1\%) of the study subjects became sputum negative for AFB, one month after initiation of Antitubercular therapy. Rest 10 patients became sputum negative at 2 month follow -up. All these 10 patients had cavitary lesion in X-ray chest at initial visit. Most of the patients (98\%) with positive X-ray finding showed radiological improvement after 2 months of antitubercular treatment. All the patients completed their therapy without any interruption up to 6 months.

Table-IV

Sputum for AFB (Acid fast bacilli) positivity among study subjects

\begin{tabular}{lc} 
Sputum positive & $\mathrm{N}(\%)$ \\
\hline One sample & $7(9.7)$ \\
Two sample & $12(16.7)$ \\
Three sample & $53(73.6)$ \\
\hline
\end{tabular}




\section{Discussion:}

The majority of our patients, 68 (94.44\%) were 30-75 years of age, similar to other studies, where most of the patients were above 40 years of age. ${ }^{11,12}$ In the present study, all patients had type $2 \mathrm{DM}$. Though the presenting symptoms of PTB is not modified with DM, the latter can affect the radiological features both in location and type of lesion. ${ }^{13,14}$ Association between diabetes mellitus and tuberculosis and their synergistic role in causing human disease has been recognized for centuries. Either diabetes mellitus (DM) is predisposing the reactivation of PTB or DM may be caused by PTB due to insulin resistance. ${ }^{15}$ In our study most of the patients were males 46 (63.9\%) which is also observed by Perez-Guzman et al. ${ }^{16}$ It is commonly seen that PTB is having high frequency in males as compared to females and this may be because of higher exposure of males or underdiagnosis of tuberculosis in females. But this male predominance in tuberculosis patients is reversed as age progressed. ${ }^{19}$ In this study, most of the PTB patients (77.3\%) do not show any growth of organism in association will mycobacterium. About 95\% of this study subjects have chest X-ray suggestive of TB. Radiological signs of PTB are more pronounced in diabetics. ${ }^{17}$ In our study cavitation was found in $34(47.2 \%)$, opacity in 29(40\%) and cavitation with opacity in 4(5.5\%) of subjects. In patients with PTB and diabetes of all ages, frequency of cavitation is high, whereas lower lobe/ lung field involvement was the frequent change. ${ }^{18}$ Most of the study (98\%) subjects showed radiological improvement within a period of 2 months. Subjects who become sputum negative after 2 months follow up had cavitary lesion at initial visit. These patients probably require antitubercular therapy for longer period and this need to be confirmed by long time study with large population.

\section{Conclusion:}

Pulmonary cavity and opacity is the commonest radiological observation among study subjects. Most of them had high ESR. Klebsiella was the commonest associated organism in sputum of PTB patients. Sputum AFB is a good diagnostic tool for PTB in diabetic subjects. Subjects with cavitary lesion require more time to become sputum negative.

\section{References:}

1. Hu FB, Manson JE, Stampfer MJ, et al. Diet, lifestyle, and the risk of type 2 diabetes mellitus in women. $\mathrm{N}$ Engl $\mathrm{J}$ Med. 2001;345:790-97.
2. Mozaffarian D, Kamineni A, Carnethon M, Djousse L, Mukamal KJ, Siscovick D. Lifestyle risk factors and newonset diabetes mellitus in older adults: the cardiovascular health study. Arch Intern Med. 2009;169:798-807

3. Stevenson CR, Critchley JA, Forouhi NG, et al. Diabetes and the risk of tuberculosis: a neglected threat to public health? Chronic Illn. 2007;3:228-45.

4. Leung CC, Lam TH, Chan WM, et al. Lower risk of tuberculosis in obesity. Arch Intern Med. 2007;167:1297-304.

5. Boutayeb A. The double burden of communicable and non-communicable diseases in developing countries.Trans R Soc Trop Med Hyg.2006;100:191-99

6. Jeon CY, Murray MB. Diabetes mellitus increases the risk of active tuberculosis: a systematic review of 13 observational studies. PLoS Med. 2008;5:e152.

7. Leung CC, Lam TH, Chan WM, et al. Diabetic control and risk of tuberculosis: a cohort study. Am J Epidemiol. 2008;167:1486-94.

8. Bloomgarden ZT. Inflammation and insulin resistance.Diabetes Care. 2003;26:1922-26.

9. Al-Tawfiq JA, Saadeh BM. Radiographic manifestations of culture-positive pulmonary tuberculosis: cavitary or non-cavitary? Int J Tuberc Lung Dis. 2009;13:367-70.

10. Guler M, Unsal E, Dursun B, Aydln O, Capan N. Factors influencing sputum smear and culture conversion time among patients with new case pulmonary tuberculosis. Int J ClinPract. 2007;61:231-35.

11. Khan SU, Cheema TM, Tariq M, Siddiqui MJ.A comparative study of radiological pattern of pulmonary tuberculosis in diabetics versus non diabetics.Pakistan Journal of Medical Research 1997; 36 (2).

12. Rizvi N, Shah RH, Inayat N, Hussain N. Differences in clinical presentation of pulmonary tuberculosis in association with age. JPMA Aug 2003; 53 (8): 321-4.

13. KossiiluE, Karachunskii MA, Kaminskaia GO, Cher-nykh NA, Zahukovskaia DE.Pulmonary tuberculosis inpatients with different types of diabetes mellitus.ProblTuberk 2002; (5): 21-4.

14. Bacakoglu F, Basoglu OK, Cok G, Sayiner A, Ates M. Pulmonary tuberculosis in patients with diabetes mellitus. Respiration. 2001; 68 (6): 595-600.

15. Kant L. Diabetes Mellitus-Tuberculosis: The brewing double trouble. Indian Journal of Tuberculosis (Editorial) 2003; 50 (4): 183-4.

16. Perez-Guzman C, Vargas MH, Torres-Cruz A, Perez-Padilla JR, Furuya ME, Villarreal-Velarde H. Diabetes modifies the male: female ratio in pulmonary tubercu-losis. Int $\mathrm{J}$ Tuberc Lung Dis. 2003 Apr; 7 (4): 354-8.

17. Mboussa J, Monabeka H, Kombo M, Yokolo D, YokaMbio A, Yala F. Course of pulmonary tuberculosis in diabetics. Rev PneumolClin. 2003 Feb; 59 (1): 39-44.

18. Umeki S, Soejima R, Hara Y. Age-dependent altera-tions in clinical features of pulmonary tuberculosis. Kekkaku 1992; 67 (1): 9-18. 